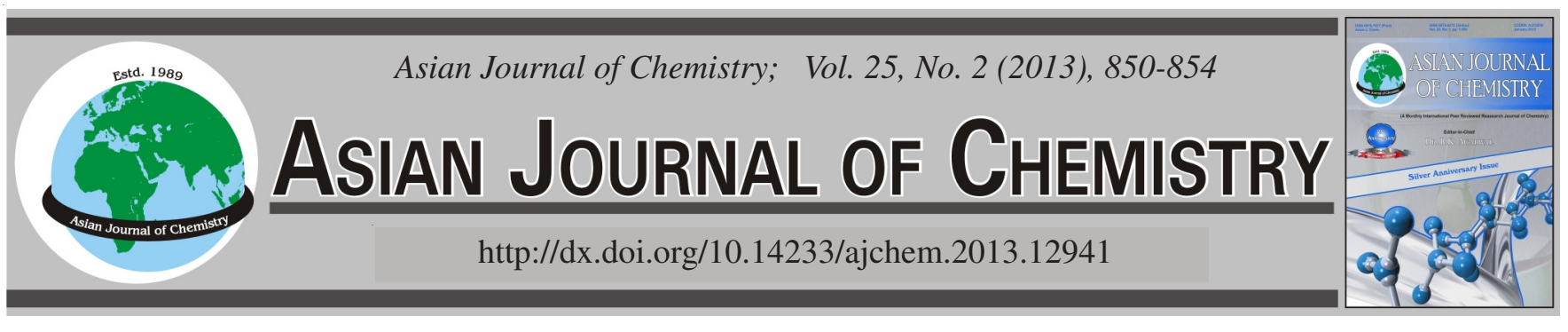

\title{
Studies on Graft Copolymerization of 2-Acrylamido-2-methylpropanesulfonic Acid onto Protein Initiated by Ammonium Persulfate
}

\author{
Mohammad Sadeghi ${ }^{*}$, Fatemeh Shafiei and Esmat Mohammadinasab
}

Department of Chemistry, Science Faculty, Arak Branch, Islamic Azad University, Arak, Iran

*Corresponding author: Fax: +98 861 3670017; Tel: +98 916 1613256; E-mail: m-sadeghi@iau-arak.ac.ir

The protein, collagen, has been chemically modified by graft copolymerization of 2-acrylamido-2-methylpropanesulfonic acid (AMPS) in an aqueous medium using ammonium persulfate as an initiator under argon atmosphere. A plausible reaction mechanism of grafting has been suggested. Evidence of grafting was obtained by comparison of FTIR spectra of collagen and homopolymer-free collagen-gpoly(2-acrylamido-2-methylpropanesulfonic acid) as well as solubility characteristics and gravimetric analysis of the products. Evidence of grafting was obtained by comparing FTIR, thermogravimetric curves of collagen and the graft copolymer as well as solubility characteristics of the products. The synthetic conditions were systematically optimized through studying the influential factors including temperature, concentration of the initiator, 2-acrylamido-2-methylpropanesulfonic acid monomer and collagen substrate.

Key Words: Graft copolymer, Collagen, 2-Acrylamido-2-methylpropanesulfonic acid.

\section{INTRODUCTION}

Graft copolymerization of hydrophilic vinyl monomers is a well-known technique employed by polymer chemists for significantly modifying the chemical and physical properties of the synthetic or natural starting materials with minimum degradation of the original properties ${ }^{1-5}$. Graft copolymers are prepared by first generating free radicals on the polysaccharide backbone and then allowing these radicals to serve as macroinitiators for the vinyl monomers. These biodegradable and low cost graft copolymers, with new properties can be used in many applications such as textiles, paper industry, agriculture, medical treatment, in petroleum industry as flocculants and thickening agents ${ }^{2,3}$ and also development of selective permeable membranes ${ }^{6}$, sorption agents ${ }^{7}$ and in fabrication of drug delivery systems ${ }^{8,9}$.

Proteins are widely distributed in nature and are synthesized mainly in animals, i.e. collagen, keratin, gelatin, etc. and in a few plants such as Soya. In general, proteins are high molecular weight polymers and their solubility in aqueous solutions is difficult. Two efficient methods for preparation of aqueous soluble proteins are alkaline and enzymatic hydrolysis. According to the literature survey, a few studies have been reported in the case of graft copolymer based on protein $^{10-12}$.

Though much work has been reported on the grafting of 2-acrylamido-2-methylpropanesulfonic acid onto various polysaccharides. Therefore, The present investigation deals with the detailed study of some major factors which affect graft copolymerization of 2-acrylamido-2-methylpropanesulfonic acid (AMPS) onto collagen, initiated by ammonium persulfate (APS) in aqueous medium with a view to elucidate the grafting mechanism.

\section{EXPERIMENTAL}

Hydrolyzed collagen (Parvar Novin-E Tehran Co.) was industrial grade which is available in market and has nearly $25 \%$ insoluble phosphate salt. 2-Acrylamido-2-methylpropanesulfonic acid (Merck, Darmstadt, Germany) and ammonium persulfate (Fluka, Buchs, Switzerland) were of analytical grade and used without further purification.

Graft copolymerization procedure: A pre-weighed amount of hydrolyzed collagen (0.5-2.5 g) was dissolved in $50 \mathrm{~mL}$ degassed distilled water and filtered to remove its insoluble salt. The solution was added to a $100 \mathrm{~mL}$ three-neck reactor equipped with a mechanical stirrer (RZR 2021, a threeblade propeller type, Heidolph, Schwabach, Germany) and the reactor was immersed in a thermostated water bath preset at a desired temperature $\left(35-65^{\circ} \mathrm{C}\right)$. Then, a given amount of monomer, AMPS (1-5 g), was added to the flask and the mixture was stirred for $10 \mathrm{~min}$. Then the initiator solution (0.01-0.05 g APS in $5 \mathrm{~mL} \mathrm{H}_{2} \mathrm{O}$ ) was added to the mixture and continuously stirred for certain times (30-120 min). An inert gas (argon) was gently bubbled into the reactor to remove the 
oxygen during the graft copolymerization reaction. The product was then worked up with methanol $(200 \mathrm{~mL})$ and dried in oven at $50{ }^{\circ} \mathrm{C}$ for $5 \mathrm{~h}$.

Homopolymer extraction: The graft copolymer, collagen$g$-poly(AMPS), was freed from poly(AMPS) homopolymer, by pouring $0.30 \mathrm{~g}$ of the product in $50 \mathrm{~mL}$ of dimethyl formamide solution. The mixture was stirred gently at room temperature for $48 \mathrm{~h}$. After complete removal of the homopolymer by filtration of the collagen- $g$-poly(AMPS), copolymer, the product was washed with methanol and dried in oven at $50{ }^{\circ} \mathrm{C}$ to reach a constant weight ${ }^{13}$.

The infrared spectra were taken on an ABB Bomem MB100 FTIR spectrophotometer. Thermogravimetric analyses were performed on a Universal V4.1D TA Instruments (SDT Q600) with 8-10 mg samples on a platinum pan under nitrogen atmosphere. Experiments were performed at a heating rate of $10{ }^{\circ} \mathrm{C} / \mathrm{min}$ until $600{ }^{\circ} \mathrm{C}$.

Grafting parameters: The grafting parameters, i.e. grafting ratio $(\mathrm{Gr} \%)$, add-on value (Ad \%) and homopolymer content $(\mathrm{Hp} \%)$, used to characterize the nature of the copolymer are defined and calculated using the following equations $^{14-17}$ :

$$
\begin{aligned}
& \mathrm{Gr} \%=100\left(\mathrm{~W}_{2}-\mathrm{W}_{0}\right) / \mathrm{W}_{0} \\
& \text { Ad } \%=100\left(\mathrm{~W}_{2}-\mathrm{W}_{0}\right) / \mathrm{W}_{2} \\
& \mathrm{Hp} \%=100\left(\mathrm{~W}_{1}-\mathrm{W}_{2}\right) / \mathrm{W}_{1}
\end{aligned}
$$

where $\mathrm{W}_{0}, \mathrm{~W}_{1}$ and $\mathrm{W}_{2}$ are the weight of the initial substrate, total product (copolymer and homopolymer) and pure graft copolymer (after DMF extraction), respectively.

\section{RESULTS AND DISCUSSION}

Grafting mechanism: A general reaction mechanism for graft copolymerization of AMPS onto collagen backbones in the presence of APS is shown in Scheme-I. In the first step, the thermally dissociating initiator, i.e. APS, is decomposed under heating $\left(65^{\circ} \mathrm{C}\right)$ to produce sulfate anion-radicals. Then, the anion-radicals abstract hydrogen from one of the functional groups (i.e. $\mathrm{COOH}, \mathrm{SH}, \mathrm{OH}$ and $\mathrm{NH}_{2}$ ) in side chains of the collagen backbones to form corresponding macro-initiators. These macroradicals initiate grafting of AMPS onto collagen backbones leading to a graft copolymer.

FTIR spectroscopy was used for identification of the graft copolymer. Fig. 1 shows the IR spectra of the collagen and the resulted graft copolymer. The band observed at $1658 \mathrm{~cm}^{-1} \mathrm{can}$ be attributed to $\mathrm{C}=\mathrm{O}$ stretching in carboxamide functional groups of substrate backbone (Fig. 1a). The broad band at $3600-3200 \mathrm{~cm}^{-1}$ is due to stretching of $-\mathrm{OH}$ groups of the collagen. The collagen- $g$-AMPS hydrogel comprises a collagen backbone with side chains that carry sulfate groups that are evidenced by a new characteristic absorption band at $1221 \mathrm{~cm}^{-1}$ (Fig. 1b). This peak attributed to ester sulfate stretching of AMPS. The stretching band of -NH overlapped with the $\mathrm{OH}$ stretching band of the collagen portion of the copolymer $^{18}$.

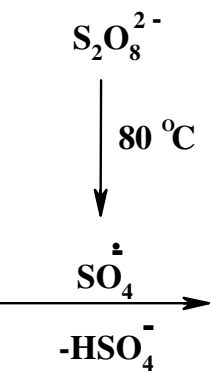<smiles>[R]C(NC(C)(C)C)C(=O)C(C)(C)C</smiles>

macroradicals

(R: various chains of 20 different amino acid)

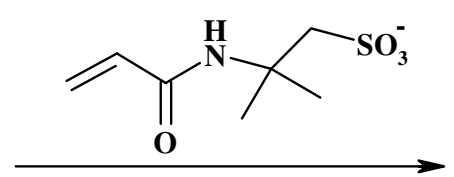

(AMPS monomer)

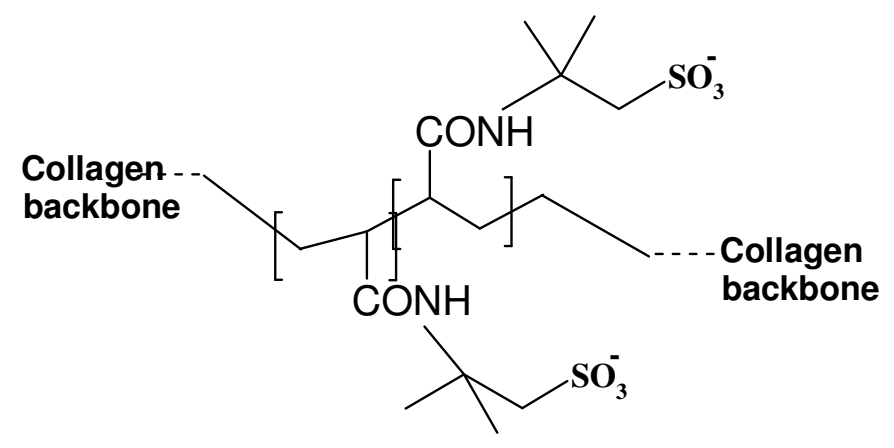

(collagen-g-AMPS copolymer)

Scheme-I: Proposed mechanistic pathway for synthesis of collagen- $g$-poly(AMPS) coplymer 


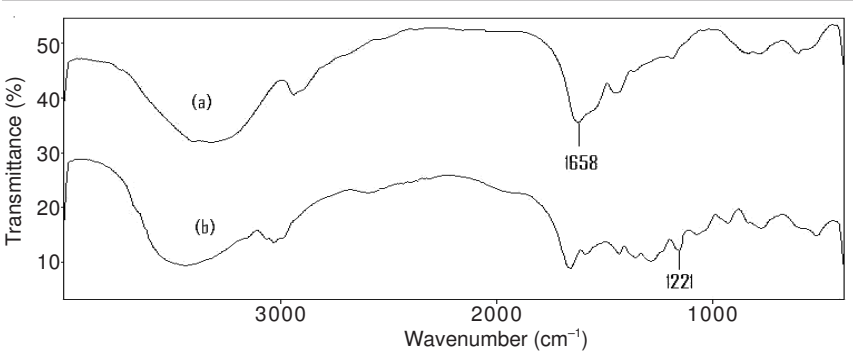

Fig. 1. FTIR spectra of collagen (a) and collagen- $g$-poly(AMPS) hydrogel (b)

Thermogravimetric analysis (TGA) was employed to thermally characterize the graft copolymer in comparison with the intact collagen (Fig. 2). The thermal stability of the grafted collagen is improved as is obvious from the TGA curve. TGA of collagen (Fig. 2a) shows a weight loss in two distinct stages. The first stage ranges between 15 and $90{ }^{\circ} \mathrm{C}$ and shows $\mathrm{ca}$. $12 \%$ loss in weight. This may correspond to the loss of adsorbed and bound water ${ }^{18}$. No such inflexion was observed in the TGA curve of collagen- $g$-AMPS. This indicated that the grafted copolymers were resistant to moisture absorption. The second stage of weight loss starts at $240{ }^{\circ} \mathrm{C}$ and continues up to $430{ }^{\circ} \mathrm{C}$ during which there was $68 \%$ weight loss due to the degradation of collagen. Grafted samples, however, show almost different behaviour of weight loss between 15 and $550{ }^{\circ} \mathrm{C}$ (Fig. 2b). The first stage of weight loss starts at $220{ }^{\circ} \mathrm{C}$ and continues up to $340^{\circ} \mathrm{C}$ due to the degradation of collagen. The second stage from 340 to $450{ }^{\circ} \mathrm{C}$ may contribute to the decomposition of different structure of the graft copolymer. The appearance of these stages indicates the structure of collagen chains has been changed, which might be due to the grafting of AMPS chains. In general, the copolymer had lower weight loss than collagen. This means that the grafting of collagen increases the thermal stability of collagen in some extent.

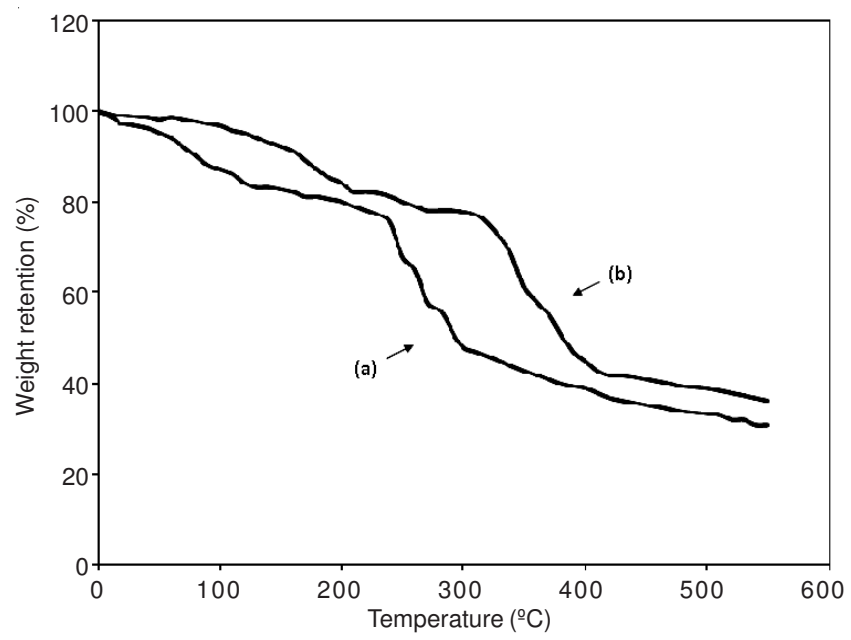

Fig. 2. TGA curves of (a) Collagen and (b) collagen-g-poly(AMPS)

Optimization of copolymerization reaction: Since polymerization variables determine the extent of grafting and homopolymer amount, certain factors affecting the grafting parameters were investigated to achieve the optimum condition of polymerization. Therefore, we optimized the grafting of 2-acrylamido-2-methyl propan sulfonic acid onto collagen in homogenous aqueous media by changing temperature, the initial concentration of monomer, initiator and the relative amount of the substrate. Within the range of the amount of the reactants used, our preliminary studies showed no considerable dependence between the reaction time and the grafting extent.

Effect of initiator concentration: Grafting of 2-acrylamido2-methylpropanesulfonic acid onto collagen backbones was carried out at various initiator concentrations $(0.01-0.07 \mathrm{~mol} / \mathrm{L})$, as shown in Fig. 3. It has been observed that the $\%$ grafting and $\%$ add-on increase initially on increasing the ammonium persulfate concentration up to $0.04 \mathrm{~mol} / \mathrm{L}$, but decrease with further increase in initiator concentration. The initial increase in $\%$ grafting and $\%$ add-on may be ascribed to the increase of the active sites on the backbone of the collagen arising from the attack of APS as a initiator.

Subsequent decrease in swelling is originated from an increase in terminating step reaction via bimolecular collision which, in turn, causes to enhance crosslinking density. In addition, the free radical degradation of collagen backbones by sulfate radical-anions is an additional reason for decrease of per cent graft copolymer at higher APS concentration. The proposed mechanism for this possibility is reported in the previous work ${ }^{19}$.

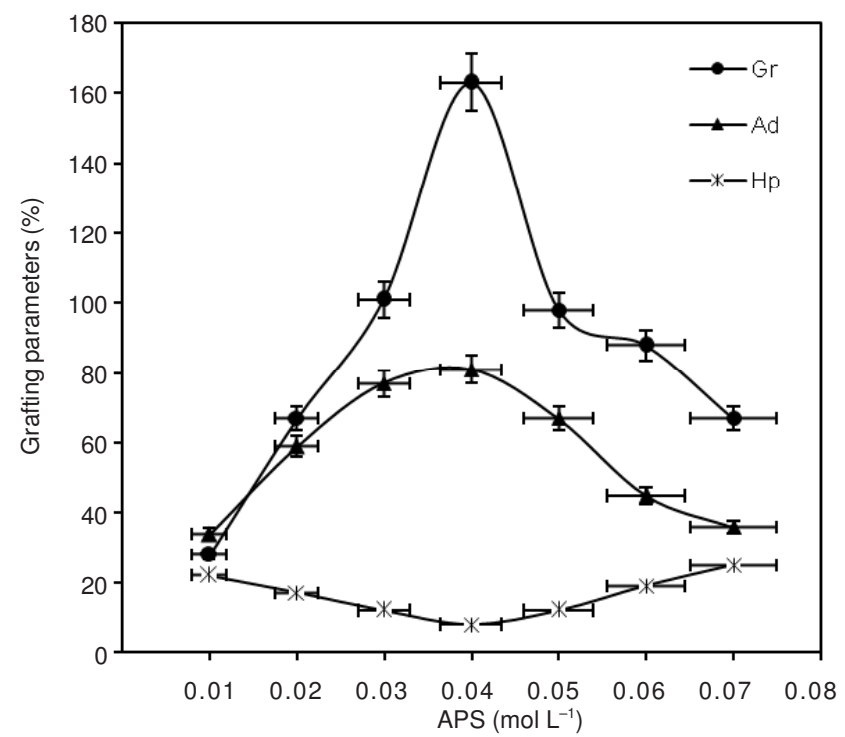

Fig. 3. Effect of initiator concentration on the grafting parameters; Reaction conditions: Collagen $2 \mathrm{wt} \%$, AMPS $0.65 \mathrm{~mol} \mathrm{~L}^{-1}$, temperature $55^{\circ} \mathrm{C}$, time $80 \mathrm{~min}$

Effect of monomer concentration: The AMPS concentration was varied from 0.2 to $1.4 \mathrm{~mol} / \mathrm{L}$ to study its effects on grafting parameters (Fig. 4). These parameters were found to be increased by enhancement of 2-acrylamido-2-methylpropanesulfonic acid concentration from 0.2 up to $0.8 \mathrm{~mol} / \mathrm{L}$. This behaviour can be attributed to the increase of monomer concentration in the vicinity of the collagen backbone and consequent greater availability and enhancement chances for molecular collisions of the reactants. The decrease in \% grafting and $\%$ add-on after a certain level of AMPS $(0.8 \mathrm{~mol} / \mathrm{L})$ is probably due to preferential homopolymerization over graft copolymerization as well as increasing the viscosity of reaction 


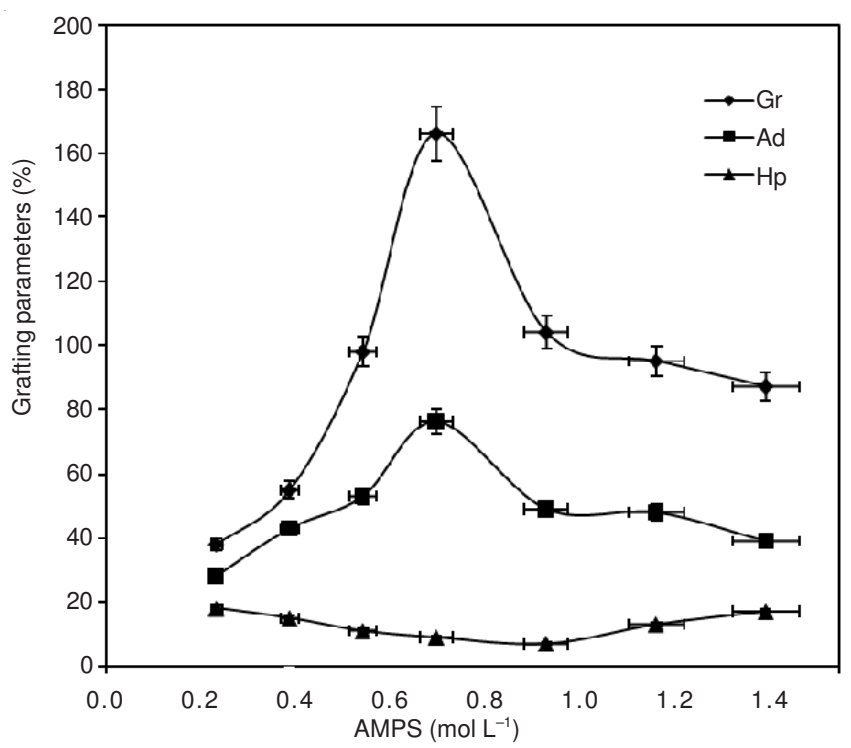

Fig. 4. Effect of the monomer concentration on the grafting parameters; Reaction conditions: Collagen 2 wt \%, APS $0.04 \mathrm{~mol} \mathrm{~L}^{-1}$, temperature $55^{\circ} \mathrm{C}$, time $80 \mathrm{~min}$

medium, which hinders the movement of free radicals. Needless to say, the increase in the chain transfer to monomer molecules may be other possible reason for the diminished grafting at higher 2-acrylamido-2-methylpropanesulfonic acid concentrations. Similar observations have been reported for the grafting of ethyl acrylate onto cellulose $e^{18,19}$ and methyl acrylate onto $\operatorname{starch}^{20}$.

Effect of collagen concentration: The results obtained by changing the collagen concentration for the graft polymerization are presented in Fig. 5. It is evident from the figure that the $\%$ grafting and $\%$ add-on increase with increase in collagen concentration up to $4 \mathrm{wt} \%$ and then decrease with further increment of protein level. The initial increase may be due to the availability of more grafting sites, where collagen can be grafted. Subsequent decrease in grafting parameters, $\%$ grafting and $\%$ add-on, in increasing collagen content more than $4 \mathrm{wt} \%$, can be explained on the basis of increase in viscosity of the medium and a decrease in the diffusion of monomers to active sites to produce graft copolymer. This observation is in close agreement with the results obtained by Zhang and $\operatorname{Tan}^{21}$.

Effect of reaction temperature: To study the influence of the reaction bath temperature on the grafting parameters, the grafting of 2-acrylamido-2-methyl propan sulfonic acid onto collagen was carried out at seven temperatures ranging from 40 to $100^{\circ} \mathrm{C}$. Fig. 6 exhibits the effect of polymerization temperature on the grafting parameters. Grafting percentage ( $\% \mathrm{Gr}$ ) is increased with increasing the temperature from 40 to $60^{\circ} \mathrm{C}$ and then decreased. This behaviour may be related to the mobility of reactive free radical sites. Moreover, higher temperatures increase the solubility of the reactants. However, grafting was decreased as the bath temperature was raised beyond $60{ }^{\circ} \mathrm{C}$. This can be accounted for in terms of chain radical termination at higher temperatures. Premature termination of growing chains and instability of the APS-protein complex are presumably another reasons for reduced amount of grafting beyond $60{ }^{\circ} \mathrm{C}$. At higher temperatures, the rate of

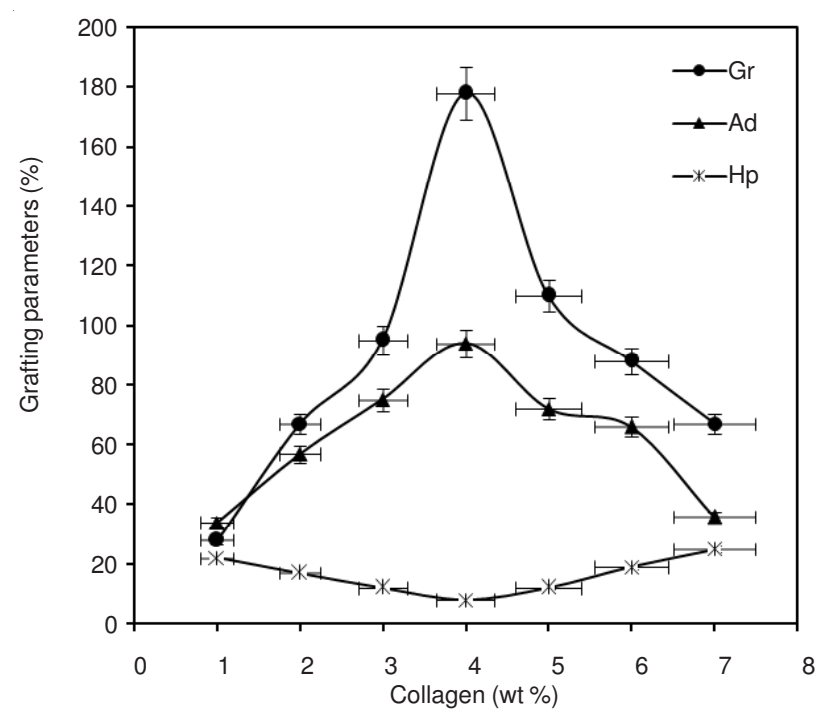

Fig. 5. Grafting parameters as functions of collagen concentration; Reaction conditions: APS $0.04 \mathrm{~mol} \mathrm{~L}^{-1}$, AMPS $0.8 \mathrm{~mol} \mathrm{~L}^{-1}$, temperature 55 ${ }^{\circ} \mathrm{C}$, time $80 \mathrm{~min}$

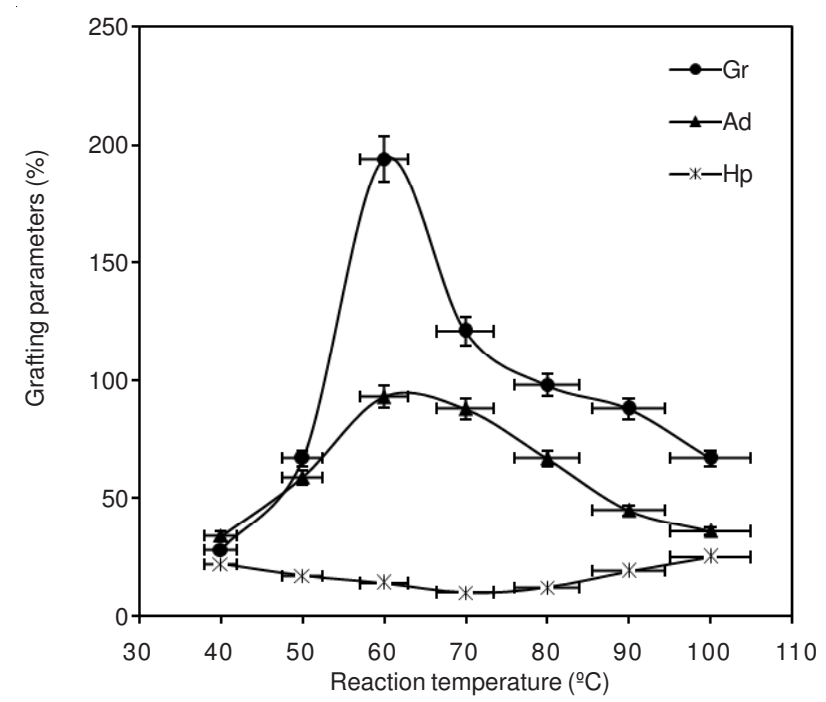

Fig. 6. Effect of the reaction temperature on the grafting parameters; Reaction conditions: Collagen 4 wt $\%$, APS $0.04 \mathrm{~mol} \mathrm{~L}^{-1}$, AMPS $0.8 \mathrm{~mol} \mathrm{~L}^{-1}$, time $80 \mathrm{~min}$

termination of the growing chain is increased and the monomer is volatilized out to some extent ${ }^{22}$.

Effect of reaction time: Grafting of 2-acrylamido-2methyl propan sulfonic acid onto collagen backbones was carried out at various polymerization times as shown in Fig. 7. The $\%$ grafting and $\%$ add-on increased with increase in the reaction time up to $90 \mathrm{~min}$ and thereafter, these parameters gradually decreased. It is obvious that the longer the reaction time, the better the graft copolymerization yield. The grafting loss may be attributed to decrease of all the consuming reactants. In addition, the decreased number of available active free radical sites for grafting and the retardation of diffusion of reactants, because of the long grafted chains at the $\mathrm{kC}$ surface, may be other possible reasons for the diminished grafting at longer reaction times. Similar time dependency of grafting parameters was reported by others ${ }^{23}$. 


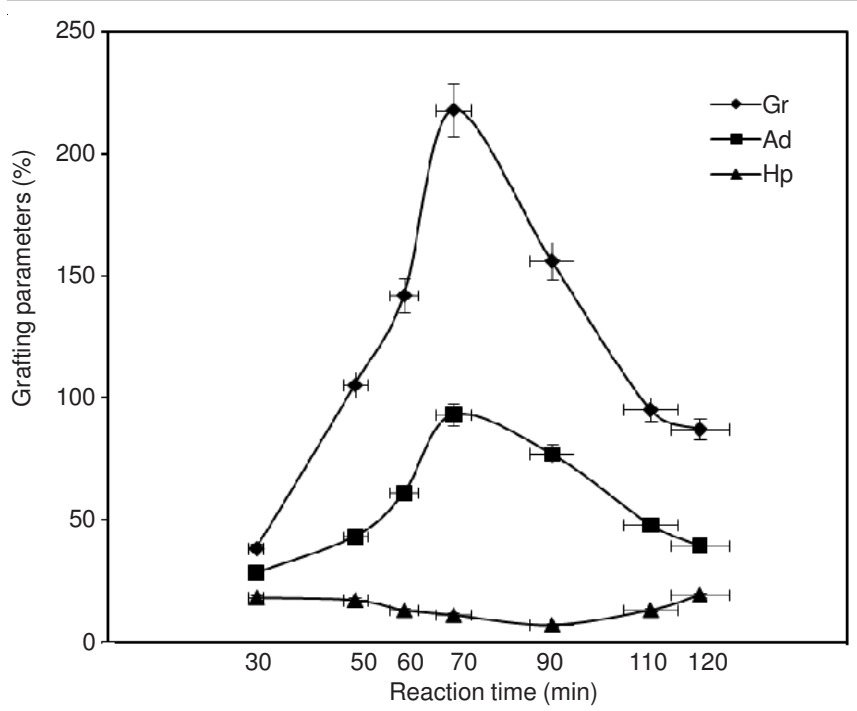

Fig. 7. Effect of the reaction time on the grafting parameters; Reaction conditions: Collagen 4 wt \%, APS $0.04 \mathrm{~mol} \mathrm{~L}^{-1}$, AMPS $0.8 \mathrm{~mol} \mathrm{~L}^{-1}$, temperature $60{ }^{\circ} \mathrm{C}$

\section{Conclusion}

The monomer, 2-acrylamido-2-methylpropanesulfonic acid (AMPS), can be easily graft copolymerized onto collagen using ammonium persulfate (APS) as an initiator in aqueous medium. In order to prove that 2-acrylamido-2-methylpropanesulfonic acid molecules were grafted, FTIR spectroscopy, TGA analysis were used. The reaction conditions were attempted to optimize for obtaining graft copolymers with higher grafting parameters. So, the reaction conditions for achieving the maximum \% Gr (218) and \% Ad (93) were found to be as follows: APS $0.04 \mathrm{~mol} / \mathrm{L}$, AMPS $0.8 \mathrm{~mol} / \mathrm{L}$, collagen $4 \mathrm{wt} \%$, reaction temperature $60^{\circ} \mathrm{C}$ and reaction time $70 \mathrm{~min}$.

\section{REFERENCES}

1. M.M. Ibrahim, E.M. Flefel and W.K. El-Zawawy, J. Appl. Polym. Sci., 84, 2629 (2002).

2. E.M. Flefel, M.M. Ibrahim, W.K. El-Zawawy and A.M. Ali, Polym. Adv. Technol., 13, 541 (2002).

3. A.A. Berlin and V.N. Kislenco, Prog. Polym. Sci., 17, 765 (1992).

4. A. Pourjavadi and M.J. Zohuriaan-Mehr, Starch/Starke, 54, 140 (2002).

5. C.D. Doyle, Anal. Chem., 33, 77 (1961).

6. V.D. Athawale and V. Lele, Starch/Starke, 52, 205 (2000).

7. D.W. Jenkins and S.M. Hudson, Chem. Rev., 101, 3245 (2001).

8. G.F. Fanta, Block and Graft Copolymerization, Wiley, London (1973).

9. A. Hebeish and J.T. Guthrie, The Chemistry and Technology of Cellulosic Copolymers, Springer-Verlag, Berlin (1981).

10. J. Huang and W. Xu, Appl. Surf. Sci., 256, 3921 (2010).

11. H. Hosseinzadeh, Asian J. Chem., 24, 2537 (2012).

12. P.K. Pandey, A. Srivastava, J. Tripathy and K. Behari, Carbohyd. Polym., 65, 414 (2006).

13. J.M. Joshi and S.V. Kumar, Polymer, 47, 2198 (2006).

14. S.J. Metz, W.J.C. Van de Ven and J. Potreck, J. Membr. Sci., 251, 29 (2005).

15. J.H. Ye, J.J. Dong, J.L. Lu, X.Q. Zheng, J. Jin, H. Chen and Y.R. Liang, Carbohyd. Polym., 81, 441 (2010).

16. I. Silva, M. Gurruchaga and I. Goñi, Carbohyd. Polym., 76, 593 (2009).

17. B. Singh, N. Chauhan and S. Kumar, Carbohyd. Polym., 73, 446 (2008).

18. G. Mino and S. Kaizerman, J. Polym. Sci., 1, 242 (1958).

19. G. Fu, J. Zhao, H. Yu, L. Liu and B. He, React. Funct. Polym., 67, 442 (2007).

20. P. Lv, Y. Bin, Y. Li, R. Chen, X. Wang and B. Zhao, Polymer, 50, 5675 (2009).

21. L.-M. Zhang and Y.-B. Tan, Macromol. Mater. Eng., 280/281, 59 (2000).

22. A. Pourjavadi, M.J.Zohuriaan-Mehr, A. Pooraghaberar and H. Hosseinzadeh, J. Polym. Mater, 21, 351 (2004).

22. R.E. Kirk and D.F. Othmer, Encyclopedia of Chemical Technology, John Wiley \& Sons, New York, edn. 4 (1992). 